\title{
Real-space fluorescence recovery after photo-bleaching of concentrated suspensions of hard colloidal spheres
}

\author{
Nikoleta B. Simeonova and Willem K. Kegel* \\ Van't Hoff Laboratory for Physical and Colloid Chemistry, Debye Institute, \\ Utrecht University, Padualaan 8, 3584 CH, Utrecht, The Netherlands. \\ E-mail: W.K.Kegel@chem.uu.nl
}

Received 8th May 2002, Accepted 10th June 2002

First published as an Advance Article on the web 18th September 2002

In concentrated suspensions of fluorescent colloidal hard spheres (close to and above the glass transition density), we bleached part of the system in cube shaped regions using high intensity laser light. Recovery of these bleached cubes was followed in real space using confocal scanning laser microscopy (CSLM). This method provides mean squared particle displacements up to timescales that are three orders of magnitude beyond those available by present experimental techniques. We show that, above the (hard sphere) glass transition density, particles move over distances of the order of their own diameter on timescales of $10^{6}$ to $10^{8}$ Brownian times. Moreover, the mean squared displacement, $\left\langle x^{2}\right\rangle$, shows powerlaw behavior over seven time $(\tau)$ decades: $\left\langle x^{2}\right\rangle \propto \tau^{(0.30 \pm 0.05)}$. This behavior is different from earlier observations by dynamic light scattering. It is argued that these differences are caused by gravity effects, as the only difference between the systems is the buoyant mass of the colloids.

\section{Introduction}

Colloidal hard spheres are extensively studied experimental model systems that exhibit two fundamental transitions. The first is a thermodynamic (first-order) phase transition from a fluid to a face centered cubic (fcc) crystal upon increasing the volume fraction, $\phi$, of the spheres. ${ }^{1-3}$ This freezing transition takes place at $\phi=0.494$. Between $\phi=0.494$ and 0.545 , fluid and crystal coexist, and at higher densities a single-phase crystal is stable. The second transition occurs when a colloidal hard sphere fluid is rapidly quenched (by centrifugation) to densities above approximately $\phi=0.575$ : in that case the system is a colloidal glass. ${ }^{3}$ This is where large-scale particle diffusion and crystallization by homogeneous nucleation stop, at least under normal gravity conditions. ${ }^{4}$ Note that this volume fraction is well below the volume fraction $\phi=0.64$ corresponding to random close packing. The nature of this glass transition is still in dispute. For example, the experimentally observed slowing down of particle diffusion at increasing volume fraction above the melting transition, and the arrest of structural relaxation (on experimental timescales) at the glass transition, can be described very well by mode coupling theory (MCT) ${ }^{5,6}$ It has recently been observed, however, that dense colloidal fluids contain distinct regions where particles move cooperatively, so-called dynamical heterogeneities. ${ }^{7,8}$ These heterogeneities were also found in molecular systems, both experimentally, ${ }^{9}$ and by computer simulation. ${ }^{10}$ Dynamical heterogeneity causes a non-Gaussian distribution of particle displacements, the magnitude of which is underestimated by MCT. Moreover, a few years ago, the notion that homogeneous nucleation stops in 
colloidal glasses was challenged: observations under microgravity showed rapid crystallization of samples with high volume fractions which fail to crystallize even after a year on earth. ${ }^{11}$

In this work we address the following questions:

1. How far do particles, on average, move in a colloidal glass? The fundamental timescale in colloidal systems is the Brownian time, $\tau_{\mathrm{B}}$, defined as the time it takes for an infinitely dilute particle to diffuse over its own radius, i.e.,

$$
\tau_{\mathrm{B}}=R^{2} / 6 D_{0}
$$

where $D_{0}$ is the diffusion coefficient at infinite dilution, being of the order of $10^{-13}-10^{-12} \mathrm{~m}^{2} \mathrm{~s}^{-1}$, depending on the solvent viscosity. In concentrated systems, the mean squared displacement, $\left\langle r^{2}\right\rangle$, of the particles as a function of time, $\tau$, can be distinguished into three regimes. At small times (but already at Brownian timescales) where virtually no interparticle collisions have occurred, diffusive behavior is observed, i.e., $\left\langle r^{2}\right\rangle=6 D_{\mathrm{s}} \tau$, where $D_{\mathrm{s}}$ is the short-time diffusion coefficient. At intermediate times, particles are caged by their neighbors and $\left\langle r^{2}\right\rangle$ as a function of time reaches a plateau: $\left\langle r^{2}\right\rangle$ is almost constant as a function of time. At long times, particles have escaped these cages and again behave diffusively, i.e., $\left\langle r^{2}\right\rangle=6 D_{\mathrm{L}} \tau$, but now the proportionality factor between mean squared displacement and time contains the long-time diffusion coefficient, $D_{\mathrm{L}}$. At densities above the glass transition, systems apparently do not reach this long-time regime, at least not on experimental timescales. On the other hand, the 'plateaus' that are observed above the glass transition density are not entirely flat: $\left\langle r^{2}\right\rangle$ keeps increasing with time (albeit more slowly than proportional) up to at least the order of $10^{5}$ Brownian times. As the formation of a critical crystal nucleus is expected to require rearrangements of particles over roughly their own diameter, the question is simply: do they get that far? At least after $10^{5}$ Brownian timescales, and just above the glass transition density $(\phi=0.59)$, particles have only moved about half their radius, as can be inferred from ref. 12. So, in principle, (Brownian) timescales that are at least several orders of magnitude longer than in ref. 12 are required to answer the question as to how far colloids move in a glass. We will show that over time intervals of the order of $10^{6}-10^{8}$ Brownian times, particles move over distances comparable to their own diameter.

2. If the relation between $\left\langle r^{2}\right\rangle$ and $\tau$ is not strictly constant, then what is it? In other words: if we define a 'glass' as a quenched liquid where long-time self diffusion is no longer observed, then, for hard spheres, there are two possibilities. The first is that, analogous to molecular systems, the glass transition corresponds to stretching of timescales beyond an experimental time window. ${ }^{13}$ The second possibility is that it is a transition that couples to a different (relaxation) behavior. In the first scenario, long-time diffusion would eventually be recovered, albeit at times that may be beyond experimental observation times. In the second case, long-time behavior would be different from diffusive ('diffusive' being defined as $\left.\left\langle r^{2}\right\rangle \propto \tau\right)$. A dramatic example is a system of hard spheres that is quenched to random close packing: in that case each sphere is caged by its neighbors, and $\left\langle r^{2}\right\rangle(\tau)$ remains essentially flat. This, in fact, is the ultimate MCT scenario: a dynamic singularity.

In order to answer these questions, we need to go to very long times. 'Classical' experimental techniques are inappropriate. For example, the largest time window that can currently be reached using dynamic light scattering (DLS) is several hours. For particles with radius $R$ of order $0.1 \mu \mathrm{m}$, this corresponds to Brownian timescales of the order of $10^{5}$. Tracking individual particles by confocal microscopy is limited by the fact that particles 'escape' the sampling volume and by hard disk capacity. In practice only much narrower time windows are available using this technique in comparison with DLS.

In this work we reach timescales that are three orders of magnitude beyond those available by DLS. Fluorescent colloidal (hard sphere) particles are irreversibly bleached in a well defined pattern, and the sample is imaged using confocal scanning laser microscopy. Subsequently the fluorescent intensity profiles of the bleached pattern are analyzed at different times after bleaching. The detailed experimental set-up is presented in the Experimental section. The method is similar in principle to 'confocal fluorescence recovery after photo-bleaching. ${ }^{14}$ This method is used in the biophysics field to determine diffusion coefficients of (diluted) proteins, see, e.g., ref. 15. The procedure is analogous to hole burning studies (see, e.g., ref. 16) and 'classical' fluorescence recovery after photo-bleaching (FRAP; see, e.g., ref. 17), but in real space. It should be noted that 'classical' FRAP suffers from similar limitations as DLS. 


\section{Experimental}

\section{System and instrumental set-up}

The model system that we used was NBD (4-methylaminoethylmethacrylate-7-nitrobenzo-2-oxa1,3-diazol) labeled polymethylmethacrylate (PMMA) particles stabilized with poly(12-hydroxystearic acid), where the fluorescent dye was incorporated during the polymerization reaction with the method described in ref. 18. The diameter of the particles is $448 \mathrm{~nm}$ and their polydispersity is $5 \%$. The particles were dispersed in a mixture of $c i s$-decalin, tetralin and carbon tetrachloride with volume ratios 31.5:36.0:32.5 as in ref. 19. In this mixture, the particles behave as hard spheres and are almost density- and refractive index matched. They bleach rapidly at maximum laser intensity ( $2 \mathrm{~mW}, \lambda=488 \mathrm{~nm}$ ) but only slowly at the reduced (about $10-15 \%$ of the maximum) intensity that was used for scanning the images for analysis. The size of the particles was chosen to be small enough so that sufficiently long timescales are accessible (note that the Brownian time scales as $\tau_{\mathrm{B}} \propto R^{3}$ with $R$ the particle radius, see eqn. (1)) but large enough so that translation (on average, see later) of the particles over fractions of their sizes can be followed. Due to the relatively large polydispersity, crystallization at $\phi \geqslant 0.5$ is expected to be slow, in particular for dense systems with $\phi>0.57$. Indeed, in the most concentrated system that we studied $(\phi=0.59)$, crystalline regions could not be observed, even after several weeks.

Using a CSLM, profiles were bleached in the form of (3-dimensional) cubes with linear sizes of the order of 10 to 100 particle diameters in the focal plane and 10 diameters in thickness. Subsequently the systems were imaged, also by CSLM, by scanning several slices perpendicular to the field of gravity, at different times (from minutes to several weeks). The sample cell and sample holder set-up we used are described in ref. 19.

\section{Data analysis}

Profiles were obtained by integrating the fluorescent intensities along both perpendicular directions of the slices (perpendicular to a side of the cube), resulting in four intensity profiles. These were compared in order to check whether flow occurred, averaged, and normalized with respect to the fluorescent intensity far away from the bleached cube. Averages were taken over several slices. In the Appendix we show that this integrated, normalized intensity profile along an axis perpendicular to a side of the cube has, to a good approximation, the form given by eqn. (A2), i.e.,

$$
I(x)=\frac{1}{2}\left(1-\operatorname{erf}\left[\frac{x}{2 \sqrt{D \tau}}\right]\right)
$$

where $D$ is the diffusion coefficient and $\tau$ is the time. This equation implies that the initial intensity profile (at $\tau=0$ ) is a step function at $x=0$ with $I(x \leqslant 0)=1$ and $I(x \geqslant 0)=0$. In practice, this is not the case. Instrumental properties cause significant deviations from this 'ideal' initial profile. Probably the most important one is the finite width of the laser beam, which (for clarity) has been neglected in the derivation given in the Appendix. As the intensity profile of the laser beam is, to a good approximation, Gaussian, it can be deduced from the derivation in the Appendix that the initial profile is expected to have the same form as eqn. (2), with $D \tau$ replaced by a quantity that is related to the width of the laser beam. In Fig. 1a we show a bleached cube in a system that is randomly close packed $(\phi=0.64)$ and where particles cannot move. Its intensity profile is shown in Fig. 1b. It reflects the 'instrumental' contribution and is indeed quite well described by eqn. (2), as can be seen in the figure. This measured profile is quantitatively reproduced even weeks later, and is also indistinguishable from a profile that was bleached in a system with $\phi=0.59$ and measured after less than a minute. These observations indicate that the time development of the profile can only be caused by particles moving in and out of the bleached region.

Clearly, the part of the intensity profile where $x<0$ is more curved than described by eqn. (2). This is probably due to scattering of the laser light by the particles, causing additional bleaching. Since this effect is not detectable for $x>0$, we neglect it in our analysis and fit the data to

$$
I(x)=\frac{1}{2}\left(1-\operatorname{erf}\left[\frac{x}{2 \sqrt{b}}\right]\right)
$$


(a)
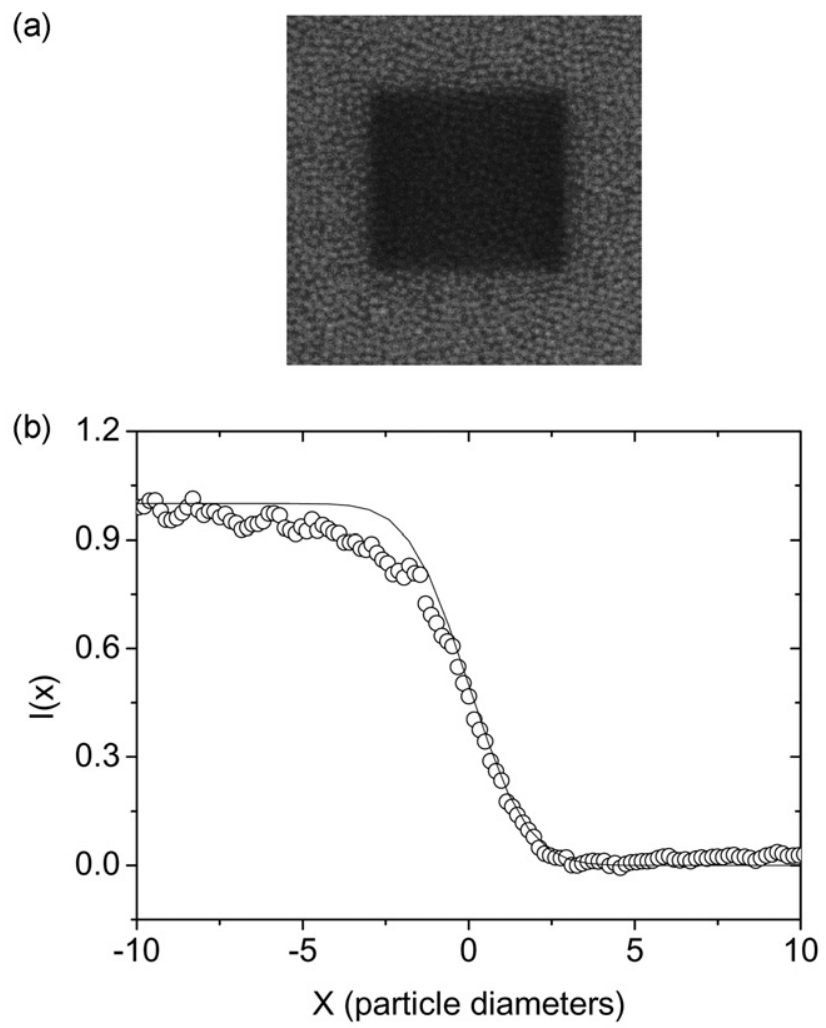

Fig. 1 (a) Bleached cube in a randomly close packed hard sphere suspension with volume fraction $\phi=0.64$. The size of the cube in the focal plane is $11 \mu \mathrm{m}$ (25 particle diameters). As only a thin slice (of 5 particle diameters) was bleached, some fluorescence is still apparent in the bleached part of the sample. The fluorescence in this region comes from particles that are out of the focal plane. (b) Normalized (horizontal) intensity profile representing the left part of the system (in fact the profile is averaged over all sides of the cube). The line is eqn. (3) with $b=0.8$ particle diameters.

where $b$ is simply related to the half width of the laser beam (if the half width is $\sigma$ then $b=\sigma \sqrt{2}$ ). The profiles for systems with $\phi<0.64$ are then described by

$$
I(x)=\frac{1}{2}\left(1-\operatorname{erf}\left[\frac{x}{2 \sqrt{D \tau+b}}\right]\right) .
$$

The values of $x$ where the fluorescent intensity is half the intensity at $x=0, x_{1 / 2}$, were corrected by subtracting the value of $x_{1 / 2}$ determined for the system with $\phi=0.64$. As also shown in the Appendix, $x_{1 / 2}$ is related to the mean squared displacement along a line, $\left\langle x^{2}\right\rangle\left(=\left\langle r^{2}\right\rangle / 3\right)$, by

$$
x_{1 / 2}^{2} \approx 0.455\left\langle x^{2}\right\rangle \text {. }
$$

\section{Results and discussion}

In Fig. 2a and b, CSLM images are shown of cube-shaped patterns at different times after bleaching, for two volume fractions, $\phi=0.45$ and $\phi=0.59$. The Brownian time is $\tau_{\mathrm{B}}=0.0263 \mathrm{~s}$ in this system. It can immediately be seen from these images that fluorescent particles move deeper into the bleached cubes as time proceeds, the process being much faster for the system with smallest volume fraction. This effect is shown quantitatively in Fig. $2 \mathrm{c}$ and $\mathrm{d}$, where the normalized intensity 
(a)

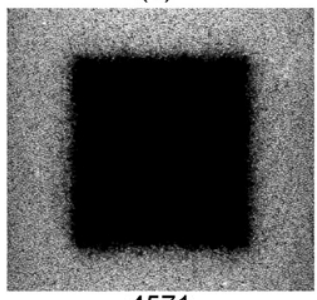

4571

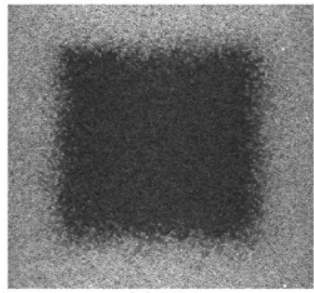

68571

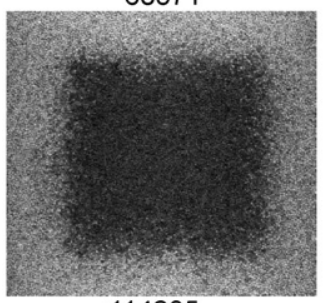

114285 (b)
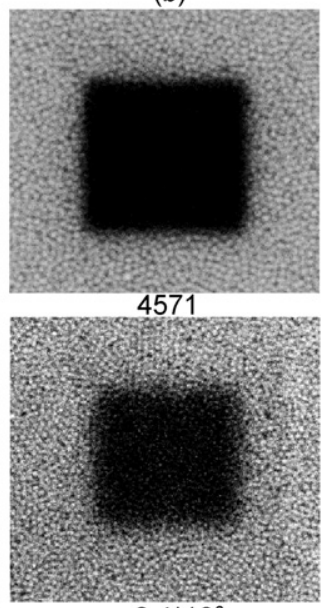

$6.4^{*} 10^{6}$

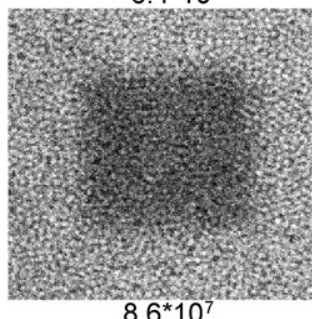

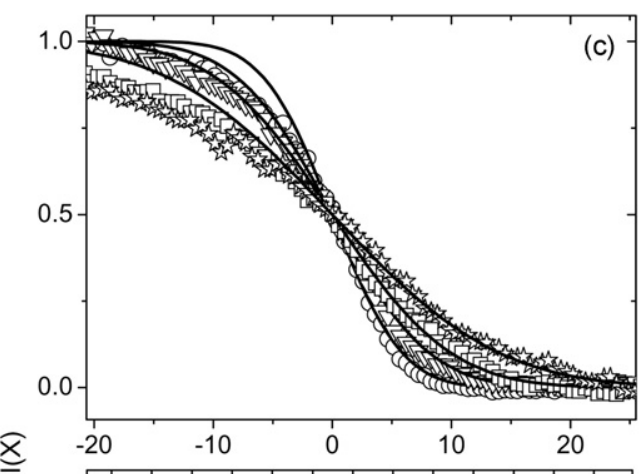

(d)

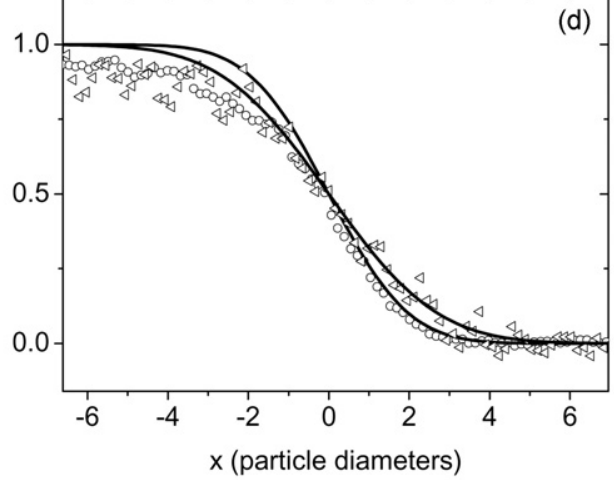

Fig. 2 (a) Bleached cubes in a colloidal hard sphere suspension with volume fraction $\phi=0.45$ at different times: 4571, 68571 and 114285 Brownian time units (indicated in the figure) where the Brownian time is $\tau_{\mathrm{B}}=0.0263 \mathrm{~s}$ for the definition see eqn. (1). The sizes of the bleached cubes in the focal plane is $65 \mu \mathrm{m}(145$ particle diameters) and their thicknesses are $3.2 \mu \mathrm{m}$. (b) Same as (a) but for a system with $\phi=0.59$ at Brownian times of $4571,6.4 \times 10^{6}$, and $8.6 \times 10^{7}$. The dimensions of the cubes are $11 \mu \mathrm{m}$ in the focal plane and thickness $5.08 \mu \mathrm{m}$. (c) Normalized fluorescence intensity profiles, $I(x)$, of the cubes shown in (a). The points correspond to 4571 (circles), 32000 (triangles), 68571 (diamonds) and 114285 (stars) Brownian times units. Corresponding lines are eqn. (4) with $2 \sqrt{D \tau+b}=6.5,9,11$, and 15.5 particle diameters. By subtracting the average intensity around the center of the cubes, data are corrected for fluorescent particles moving in from outside the cubes in the vertical direction. Averages were taken over 5 to 6 slices in a cube. (d) Same as (c), but now for the system with $\phi=0.59$ shown in (b). Circles and triangles are the normalized intensity profiles taken 4571 and $6.4 \times 10^{6}$ Brownian time units after bleaching, respectively. Lines are eqn. (4) with $2 \sqrt{D \tau+b}=2.2$ and 3 particle diameters. Averages were taken over 7 to 9 slices in a cube.

profiles are plotted together with the theoretical profiles, eqn. (4). Clearly, at $x>0$ the profiles are quantitatively described by eqn. (4) for all times, even very long ones. This is not the case for the profiles at $x<0$, probably as a result of scattering (as discussed in the previous section). However, this does not affect the analysis. In particular for the system with $\phi=0.59$ (Fig. 2d), the data are quite noisy. The reason is that much smaller cubes (linear size $11 \mu \mathrm{m}$ ) were analyzed compared to the system with $\phi=0.45$ (linear size $65 \mu \mathrm{m}$ ), resulting in a higher resolution but more noise because of (effectively) smaller numbers of particles being averaged.

The values of the (long time) diffusion coefficient, $D$, can be extracted from the values of $x_{1 / 2}$ that follow directly from the profiles in Fig. $2 \mathrm{c}$ and $2 \mathrm{~d}$. These values vary between 0.005 and 0.015 (in units of $D_{0}$ ) for $\phi=0.45$, which is compatible with the value 0.018 for $\phi=0.466$ as reported in ref. 12 but the error range is large. The values of $D$ for the system with $\phi=0.59$ are of the order of $10^{-8}$, but this value cannot be interpreted as a long-time diffusion coefficient, as it is shown in Fig. 3 that long-time diffusion (i.e., $\left.\left\langle x^{2}\right\rangle \propto \tau\right)$ is not observed for this system. 


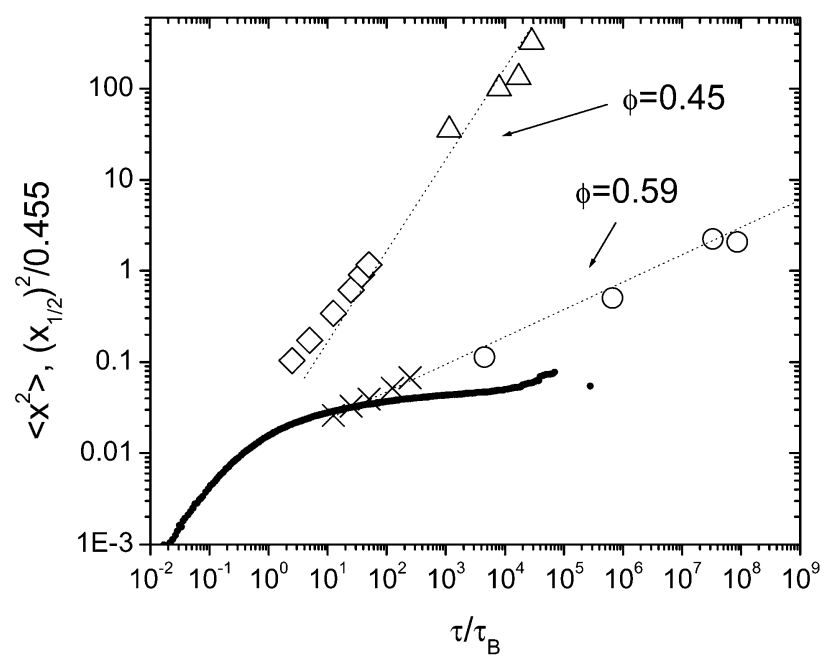

Fig. 3 Mean squared displacements $\left\langle x^{2}\right\rangle$ and $\left(x_{1 / 2}\right)^{2} / 0.455$ (as defined in the text) in units of the squared particle radius versus time in units of Brownian time $\tau_{\mathrm{B}}$. The values of $x_{1 / 2}$ that resulted from the profiles in Fig. $2 \mathrm{c}$ and $\mathrm{d}$ were corrected for the 'instrumental' contribution (of magnitude 1.66 particle radii) as shown in Fig. 1b. Triangles correspond to the system with volume fraction $\phi=0.45$ and open circles to the system with $\phi=0.59$. Diamonds and crosses are (CSLM) data reported in ref. 7 obtained from tracking individual particles with $\phi=0.45$ and 0.60 , respectively. Closed circles are data from van Megen and coworkers ${ }^{12}$ obtained with DLS for $\phi=0.59$. The upper dotted line connects the two sets of data for $\phi=0.45$ and is given by $\left\langle x^{2}\right\rangle=\left(x_{1 / 2}\right)^{2} / 0.455=0.0167\left(\tau / \tau_{\mathrm{B}}\right)$. The lower dotted line connects the datasets for $\phi=0.59$ and 0.60 from this work and the work reported in ref. 7 and is given by $\left\langle x^{2}\right\rangle=\left(x_{1 / 2}\right)^{2} / 0.455=0.012\left(\tau / \tau_{\mathrm{B}}\right)^{0.3}$.

In Fig. 3 we plotted the values of $x_{1 / 2}^{2} / 0.455$ (the dividing factor being chosen so that direct comparison with $\left\langle x^{2}\right\rangle$ is allowed, see eqn. (5)) as a function of time for the systems with volume fractions $\phi=0.45$ and 0.59 . For comparison we plotted values of $\left\langle x^{2}\right\rangle$ obtained from analyzing displacements of individual particles, as reported in ref. 7, in the same figure. We stress that although this method also makes use of CSLM, it is a different method from the one that we employed in this work. We also plotted values of $\left\langle x^{2}\right\rangle\left(=\left\langle r^{2}\right\rangle / 3\right)$ that were reported by van Megen and coworkers ${ }^{12}$ and obtained by DLS.

It can be seen in Fig. 3 that the data obtained in this work and those from ref. 7 can simply be connected by straight lines in the log-log representation. This is to be expected for the system with $\phi=0.45$ : from approximately 10 Brownian time units on, this system is in the long-time diffusion limit (see the Introduction), being characterized by a unit slope in Fig. 3. The (two) points at earlier times clearly correspond to the intermediate regime. The short-time diffusion regime cannot be sampled by our CSLM technique because of the finite scanning speed $(0.25 \mathrm{~s}$ per frame). This regime is obvious from the (DLS) data of van Megen and coworkers (up to, say, $10^{-1}$ Brownian time units, note that their data at even smaller timescales are not shown). We conclude from this result that the system with $\phi=0.45$, at long times, behaves as expected and thus that our new method for determining displacements 'works'. Although the data are somewhat scattered around the line of unit slope in Fig. 3, at least the procedure results in the expected order of magnitude for the mean squared displacements (MSD).

Now let us turn to the dense system with $\phi=0.59$. It immediately emerges from Fig. 3 that the mean squared displacements are larger, at comparable timescales, than those reported by van Megen and coworkers ${ }^{12}$ for a system with the same volume fraction. For example at $\tau / \tau_{\mathrm{B}}=4571$, we find that particles move $\sqrt{\left\langle x^{2}\right\rangle} \approx 0.34$ particle radii whereas it follows from the data of van Megen and coworkers that $\sqrt{\left\langle x^{2}\right\rangle} \approx 0.22$ particle radii (see Fig. 3). Our findings are however consistent with earlier measurements (crosses in Fig. 3) using a different method. The tendency of the MSD to increase with time (crosses in Fig. 3) is extended to the much longer timescales studied here (open circles in Fig. 3). In particular, the data are consistent with a power-law behavior: $\left\langle x^{2}\right\rangle \propto \tau^{(0.30 \pm 0.05)}$ over 7 time decades, reaching displacements of the order of a particle diameter. 
This behavior is fundamentally different from predictions by mode coupling theory. On the other hand, the DLS data from ref. 12 in Fig. 3 also show a slowly increasing MSD with time, although this increase is not as steep as observed for our data, and does not follow power-law behavior.

The only difference between the systems studied by van Megen and coworkers, and those studies here and in ref. 7, is the buoyant mass of the colloidal particles. The mass density difference between the PMMA particles and the solvent in the mixture of tetralin and decalin in ref. 12 is $\Delta \rho_{\mathrm{m}} \approx 0.3 \mathrm{~g} \mathrm{ml}^{-1}$, while in our system it is roughly an order of magnitude smaller. Therefore, a possible explanation for the much steeper increase in $\left\langle x^{2}\right\rangle$ found here is that the system of van Megen and coworkers is slowed down by gravity effects. The particles used in this work are of comparable size to those in ref. 12, and so is the solvent viscosity. The most obvious effect of gravity is sedimentation, resulting in densification of the systems. We can easily estimate the relative timescales where sedimentation effects become apparent. The timescale where particles sediment over a height $\Delta h$ is $\Delta t=\Delta h / \nu_{\text {sed }} \propto 1 / \Delta \rho_{\mathrm{m}}$, where $\nu_{\text {sed }}$ is the sedimentation velocity which is proportional to the (mass) density difference for equally sized particles. If the plateau in $\left\langle x^{2}\right\rangle$ versus $\tau$ as found by van Megen and coworkers is caused by sedimentation effects (or consequences of sedimentation effects), then the above argument implies that our data should undergo the same fate, with a delay of only a decade in time. Clearly, this is not what happens. The much steeper increase in the MSD with time that we find persists over many decades. Only the data point at $10^{8}$ Brownian time units suggests 'collapse' of the system, but this needs to be verified. Therefore we conclude that (consequences of) sedimentation does not explain the difference between our data and the DLS results. Still, we expect gravity to play a role: as mentioned in the introduction, a colloidal glass was found to crystallize rapidly under microgravity, ${ }^{11}$ and also in a density matched solvent (with $\Delta \rho_{\mathrm{m}}$ even an order of magnitude smaller than the one studied here). ${ }^{20}$ In the system studied here, the polydispersity of $5 \%$ in combination with a small gravitational field apparently suppresses crystallization. The influence of gravity is therefore expected to be more subtle and possibly is related to the build-up of stress in the system. ${ }^{21}$ An intriguing question that remains is what mechanism, on particle length scales, leads to behavior of the form $\left\langle x^{2}\right\rangle \propto \tau^{0.30}$. Recently it was shown that particles moving in one dimension ('file movement') result in $\left\langle x^{2}\right\rangle \propto \tau^{0.5} .{ }^{22}$ This behavior reflects 'single file diffusion', which implies that the displacements of the particles are highly correlated and collective

\section{Conclusion and further work}

In this work we determined the displacements of colloidal particles at very long (Brownian) timescales: three order of magnitude longer than the present state of the art. We addressed the questions as to how far particles move in a colloidal hard sphere glass, and what the relation is between the MSD and time on long timescales. It was shown that particles move over distances of the order of their own diameter on timescales of the order of $10^{8}$ Brownian times. Moreover our MSD data are consistent with $\left\langle x^{2}\right\rangle \propto \tau^{(0.30 \pm 0.05)}$ over 7 time decades. This increase is much steeper than the extrapolated behavior of MSDs measured previously by DLS. The steep increase is however consistent with earlier measurements where individual particles were tracked. We argue that gravity may be responsible for the difference between our MSD data and those obtained by DLS. We currently are setting up experiments where the density difference between the particles and solvent is systematically varied.

\section{Appendix: Mean squared displacements from fluorescence intensity profiles in partially bleached samples}

The probability of finding a (fluorescent) particle at position $x$ on a line at time $\tau$, provided that its initial position is $x_{0}$, is the canonically averaged displacement distribution (the self-van Hove correlation function) given by

$$
p\left(x, x_{0}, \tau\right)=\sqrt{\frac{1}{4 \pi D \tau}} \exp \left(\frac{-\left(x-x_{0}\right)^{2}}{4 D \tau}\right),
$$


where $D$ is the diffusion coefficient. Although this Gaussian form is not strictly correct for concentrated systems (in particular close to the glass transition), deviations from Gaussian behavior tend to disappear on the long timescales that we study (see ref. 7, 8, 23 and 24), although they may persist for long times in certain glassy states. ${ }^{25}$ However, our final result can easily be generalized to any symmetrical displacement distribution.

Now consider two half spaces. One of them, extending from $x=0$ to $\propto$, contains the bleached part of the sample. The other one, extending from $x=-\propto$ to 0 , is the unbleached part, consisting of fluorescent particles. This is the (ideal, see Experimental section) situation directly following the bleaching process (see Fig. 1a), assuming that this process is fast compared to the relaxation times of the particles, and that the width of the laser beam is small compared to the particle size. Then, the probability of finding a fluorescent particle at position $x$ after a certain time $\tau$, independent of its original position $x_{0}$ is

$$
p(x, t)=\int_{-\infty}^{0} p\left(x, x_{0}, \tau\right) \mathrm{d} x_{0}=\frac{1}{2}\left(1-\operatorname{erf}\left[\frac{x}{2 \sqrt{D \tau}}\right]\right) .
$$

In this equation, $\operatorname{erf}(z)=\frac{2}{\sqrt{\pi}} \int_{0}^{z} \mathrm{e}^{-t^{2}} \mathrm{~d} t$ is referred to as the error function. Eqn. (A2) is just the time development of the integrated (normalized) fluorescent intensity profile $I(x, \tau)$ along one of the two axes perpendicular to the sides of the cube, i.e.,

$$
I(x, \tau)=\frac{1}{I_{\infty}} \int_{\text {cube-side }} I(x, y, \tau) \mathrm{d} y=p(x, \tau),
$$

where the assignments $x$ and $y$ are arbitrary as long as they are perpendicular to one another and to a side of the cube. The intensity $I(x, \tau)$ is normalized with respect to the fluorescent intensity far away from the bleached cube $I_{\infty}$ in eqn. (A3). In the coordinate system defined above, the profile goes from 1 (at negative values of $x$ ) to 0 (at positive values of $x$ ) and equals $\frac{1}{2}$ at $x=0$ (we make use of this property in eqn. (A5)), see Fig. 1 and 2. This is always true if the bleached and unbleached volumes are equal. In general, it is a (very) good approximation if only a small fraction of the cube is recovered by fluorescent particles, as is the case in this work. The profile has some interesting properties:

(1) Its 'zeroth moment' in positive half space is proportional to the square root of time, i.e.,

$$
A(\tau)=\int_{0}^{\infty} I(x, \tau) \mathrm{d} x=\sqrt{\frac{D \tau}{\pi}} .
$$

(2) But any value of $x$ where $I(x, \tau)$ is some fraction of its value at $x=0$ is also proportional to the square root of time. In this work we use the value of $x$ where the intensity is half the intensity at $x=0$. This value of $x$ is $x_{1 / 2}$, given by

$$
1 / 2 I(x=0, \tau)=\frac{1}{4}=\frac{1}{2}\left(1-\operatorname{erf}\left[\frac{x_{1 / 2}}{2 \sqrt{D \tau}}\right]\right) .
$$

Solving numerically the equation $\operatorname{erf}\left[\frac{x_{1 / 2}}{2 \sqrt{D \tau}}\right]=\frac{1}{2}$ leads to

$$
x_{1 / 2} \approx 0.954 \sqrt{D \tau} \text {. }
$$

This result can immediately be compared to the important result that the second moment of the (Gaussian) displacement distribution, eqn. (A1) with $x_{0}=0$, is

$$
\left\langle x^{2}\right\rangle=2 D \tau
$$

so that

$$
x_{1 / 2}^{2} \approx 0.455\left\langle x^{2}\right\rangle
$$


Eqn. (A8) provides the link between earlier results arising from DLS, ${ }^{12}$ microscopy ${ }^{7,8,23}$ and computer simulation.

The numerical factors in eqn. (A6) and (A8) correspond to a Gaussian displacement distribution (eqn. (A1)). Any deviation from Gaussian behavior leads to a different time dependence of the displacements, eqn. (A6) and eqn. (A7). Such deviations will show up if the values of $x_{1 / 2}$ are compared to the values of $D \tau$ that result from fitting eqn. (A2) to the experimental intensity profiles

\section{Acknowledgements}

We thank Alfons van Blaaderen for continuing discussions and suggestions, Bill van Megen for kindly providing the DLS data, and Gijsje Koenderink for comments on the manuscript. Els de Hoog is thanked for bringing to our attention references on 'confocal FRAP' in the biophysics field.

\section{References}

1 W. W. Wood and J. D. Jacobsen, J. Chem. Phys., 1957, 27, 1207.

2 B. J. Alder and T. E. Wainwright, J. Chem. Phys., 1957, 27, 1208.

3 P. N. Pusey and W. van Megen, Nature, 1986, 320, 340.

4 W. van Megen and S. M. Underwood, Nature, 1993, 362, 616.

5 W. Gotze and L. Sjogren, Phys. Rev. A, 1991, 43, 5442.

6 W. van Megen and S. M. Underwood, Phys. Rev. Lett., 1993, 70, 2766.

7 W. K. Kegel and A. van Blaaderen, Science, 2000, 287, 290.

8 E. R. Weeks, J. C. Crocker, A. C. Levitt, A. Schofield and D. A. Weitz, Science, 2000, 287, 672.

9 M. T. Cicerone and M. D. Ediger, J. Chem. Phys., 1996, 103, 5684.

10 W. Kob, C. Donati, S. J. Plimpton, P. H. Poole and S. C. Glotzer, Phys. Rev. Lett., 1997, 79, 2827.

11 J. Zhu, M. Li, R. Rogers, W. Meyer, R. H. Ottewill, Crew Shuttle Columbia, W. B. Russel and P. M. Chaikin, Nature, 1997, 387, 883.

12 W. van Megen, T. C. Mortensen, S. R. Williams and J. Müller, Phys. Rev. E, 1998, 58, 6073.

13 C. A. Angell, Science, 1995, 267, 1924.

14 H. Kubitscheck, P. Wedekind and R. Peters, Biophys. J., 1994, 67, 946.

15 P. Gribbon and T. E. Hardingham, Biophys. J., 1998, 75, 1032.

16 O. Kircher, B. Schiener and R. Böhmer, Phys. Rev. Lett., 1998, 81, 4520.

17 A. Imhof, A. van Blaaderen, G. Maret, J. Mellema and J. K. G. Dhont, J. Chem. Phys., 1994, 100, 2170.

18 G. Bosma, C. Pathmamanoharan, E. H. A. de Hoog, W. K. Kegel, A. van Blaaderen and H. N. W. Lekkerkerker, J. Colloid Interface Sci., 2002, 245, 292.

19 E. H. A. de Hoog, W. K. Kegel, A. van Blaaderen and H. N. W. Lekkerkerker, Phys. Rev. E, 2001, 64, 021407.

20 W. K. Kegel, Langmuir, 2000, 16, 939.

21 A. J. Liu and S. R. Nagel, Nature, 1998, 396, 21.

22 Q. H. Wei, C. Bechinger and P. Leiderer, Science, 2000, 287, 625.

23 A. Kasper, E. Bartch and H. Sillescu, Langmuir, 1998, 14, 5004.

24 T. C. Mortensen and W. van Megen, in Slow Dynamics in Complex Systems, AIP Conf. Proc., 1998, 469, 3.

25 K. Vollmayr-Lee, W. Kob, K. Binder and A. Zippelius, J. Chem. Phys., 2002, 116, 5158. 